\title{
Partial electron localization in a finite-size superlattice placed in an electric field
}

\author{
K. R. Vlasov, M. A. Pyataev, A. V. Shorokhov \\ July 2, 2021 \\ Ogarev Mordovia State University, 430005, Bolshevistskaya, 68, Saransk, \\ Russia
}

\begin{abstract}
Partial electron localization in a finite-size superlattice placed in an electric field is considered. The role of electric field in forming of quasilocalized states is investigated. A quantitative criterion for the degree of partial localization is suggested based on analysis of maximal probability density of finding an electron at a given point. It is found that with increase in the electric field the degree of localization does not increase monotonically. Furthermore, the localization is affected stronger by the amplitude of superlattice potential than by the electric field.
\end{abstract}

\section{Introduction}

The problem of electron localization in a periodic potential under applied homogeneous electric field $F$ attracts the scientific interest for many years since 1960 when G.H. Wannier suggested the appearance of discrete energy spectrum for such a system [1]. Using a basis of Bloch functions and expansion of individual terms of the equation in a powers series, he concluded that the electron energy spectrum for the system consists of a set of equidistant eigenvalues separated by the energy $e F d$, where $e$ is the 
electron charge and $d$ is the period of the potential. This phenomenon was called Wannier-Stark localization. Later, the arguments of Wannier have been criticized several times [2-4]. In particular, it was proven [4] that energy spectrum of Bloch electron in an external electric field is continuous for physically meaningful regular potentials. Thus, the complete WannierStark localization is impossible. However, Wannier-Stark localization can manifest itself as a set of quasibound states or resonances [5-7]. These resonances appear in the energy dependence of various physical quantities. They have been observed in many experiments and interpreted as evidence of Wannier-Stark localization [8-11]. Nowadays the concept of WannierStark ladder still attracts much attention because it is applied for describing various phenomena in many novel systems such as graphene [12,13], optical lattices [14,15] natural $\mathrm{SiC}$ superlattices [16-18] and others.

It is obvious that from practical point of view the problem of partial electron localization in electric field is more important for the finite superlattice because every real structure has finite size.

\section{Hamiltonian and wave function}

The aim of the present work is to study the electron localization effect in a one-dimensional periodic system containing a few dozens of periods, placed in a homogeneous electric field. The superlattice in an electric field is described by the Hamiltonian

$$
\hat{H}=\frac{\hat{p}_{x}^{2}}{2 m^{*}}+U(x)-e F x,
$$

where $m^{*}$ is the electron effective mass, $\hat{p}_{x}$ is the electron momentum operator and $U(x)$ is the stepwise superlattice potential given by the following equation:

$$
U(x)= \begin{cases}U_{0}, & x-j d<a \\ 0, & x-j d>a .\end{cases}
$$




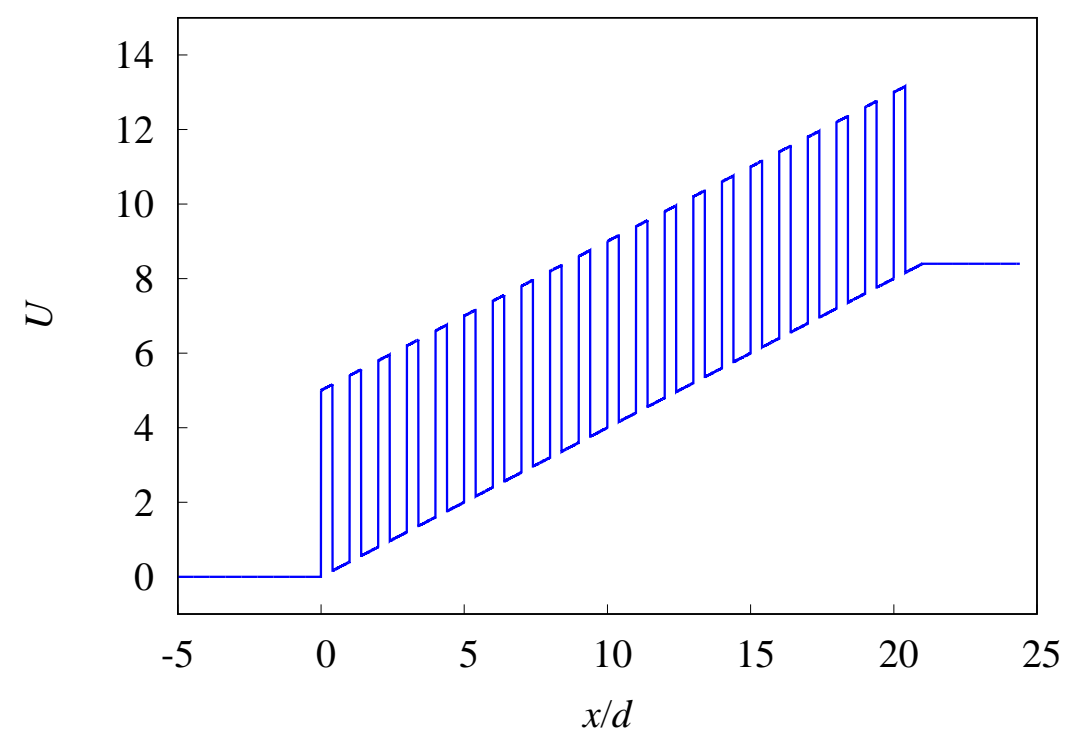

Figure 1: The potential energy for the superlattice in the electric field for $N=20$, $U_{0}=5 E_{d}, e F d=0.4 E_{d}, a=0.4 d$.

Here $a$ is the barrier width and $j=[x / d]$ is the integer part of the number $x / d$. Schematic representation of potential energy for the superlattice in the electric field is shown in Fig. 1. We assume that electric field vanishes outside the superlattice that is why the potential is taken to be constant in regions $x<0$ and $x>N d$.

Since we are focused on the role of electric field in formation of quasibound states we consider the boundary conditions which do not lead to appearance of the discrete energy spectrum in the absence of the electric field. In contrast to the problem of finding the eigenvalues for the Hamiltonian which takes place in case of rigid wall boundary conditions [19] we consider the scattering problem in which an electron wave of unit amplitude goes from the region of zero potential energy $(x \rightarrow-\infty)$ and then propagates through the superlattice or is reflected back (Fig. 1). It should be noted that propagation is possible only for energies $E>N e F d$ while in the opposite case electrons are completely reflected.

The system can be characterized by three independent parameters with 
the dimension of energy: the height $U_{0}$ of the potential barrier between the layers of superlattice, the size quantization energy $E_{d}=\hbar^{2} /\left(2 m^{*} d^{2}\right)$, and the step of the Wannier-Stark ladder $E_{F}=e F d$.

The electron wave function for $j$-th region can be represented in the form [20, 21]

$$
\psi_{j}(x)=\alpha_{j} \operatorname{Ai}\left(\xi_{j}\right)+\beta_{j} \operatorname{Bi}\left(\xi_{j}\right),
$$

where $\operatorname{Ai}(z)$ and $\operatorname{Bi}(z)$ are the Airy functions of the first and second kind respectively, $\alpha_{j}$ and $\beta_{j}$ are some coefficients. The argument $\xi_{j}$ of the Airy functions is given by

$$
\xi_{j}(x)=\left(\frac{2 m^{*}}{\hbar^{2} e^{2} F^{2}}\right)^{1 / 3}\left(e F x-E+U_{j}\right),
$$

where $U_{j}=0$ for even $j$ and $U_{j}=U_{0}$ for odd $j$.

The wave functions at neighbouring regions are related to each other via continuity boundary conditions for the wave function and its derivative. We can get the reciprocal relation from the conditions for the coefficients $\alpha_{j}$ and $\beta_{j}$

$$
\left(\begin{array}{c}
\alpha_{j+1} \\
\beta_{j+1}
\end{array}\right)=M_{j+1}^{-1}\left(x_{j+1}\right) M_{j}\left(x_{j+1}\right)\left(\begin{array}{c}
\alpha_{j} \\
\beta_{j}
\end{array}\right),
$$

where $x_{j}$ is the point separating $(j-1)$-th and $j$-th regions. The matrix $M_{j}(x)$ has the form

$$
M_{j}(x)=\left(\begin{array}{cc}
\operatorname{Ai}\left(\xi_{j}\right) & \operatorname{Bi}\left(\xi_{j}\right) \\
\operatorname{Ai}^{\prime}\left(\xi_{j}\right) & \operatorname{Bi}^{\prime}\left(\xi_{j}\right)
\end{array}\right) .
$$

The wave function in the region $x<0$ is a superposition of incident and reflected waves

$$
\psi_{0}(x)=\exp \left(i k_{0} x\right)+r \exp \left(-i k_{0} x\right)
$$

where $r$ is the reflection amplitude and $k_{0}=\left(2 m^{*} E\right)^{1 / 2} / \hbar$. The region $x>N d$ contains only propagated wave with the amplitude $t$

$$
\psi_{n}(x)=t \exp \left(i k_{n} x\right)
$$


where $n=2 N+1$ and

$$
k_{n}=\frac{\sqrt{2 m^{*}(E-N e F d)}}{\hbar} .
$$

We note that equation (8) is valid in both cases $E>N e F d$ and $E<N e F d$. However, in the second case, the wave vector $k_{n}$ has only imaginary part and $t$ can not be regarded as a transmission amplitude.

From equations (6), (7) and (8) we can get the following relation between the reflection amplitude $r$ and the transmission amplitude $t$ :

$$
\left(\begin{array}{l}
t \\
0
\end{array}\right)=S\left(\begin{array}{l}
1 \\
r
\end{array}\right)
$$

where the scattering matrix $S$ can be represented in the form

$$
S=L_{n}^{-1} M_{n-1}\left(x_{n}\right) M_{n-1}^{-1}\left(x_{n-1}\right) \ldots M_{1}\left(x_{2}\right) M_{1}^{-1}\left(x_{1}\right) L_{0}
$$

Here $L_{0}$ is given by

$$
L_{0}=\left(\begin{array}{cc}
1 & 1 \\
i k_{0} & -i k_{0}
\end{array}\right)
$$

and $L_{n}$ has the form

$$
L_{n}=\left(\begin{array}{cc}
\exp \left(i k_{n} x_{n}\right) & \exp \left(-i k_{n} x_{n}\right) \\
i k_{n} \exp \left(i k_{n} x_{n}\right) & -i k_{n} \exp \left(-i k_{n} x_{n}\right)
\end{array}\right),
$$

where $n=2 N+1$ and $k_{n}$ is given by Eq. (9). Calculating matrix $S$ and then solving Eq. (10) we can obtain transmission and reflection amplitudes and then find all the coefficients for the wave function.

\section{Results and discussion}

As a criterion of partial electron localization we consider the probability density $\rho(x)=|\psi(x)|^{2}$ of finding an electron at a given point $x$. The functions $\rho(x)$ for two different values of energy corresponding to total electron 

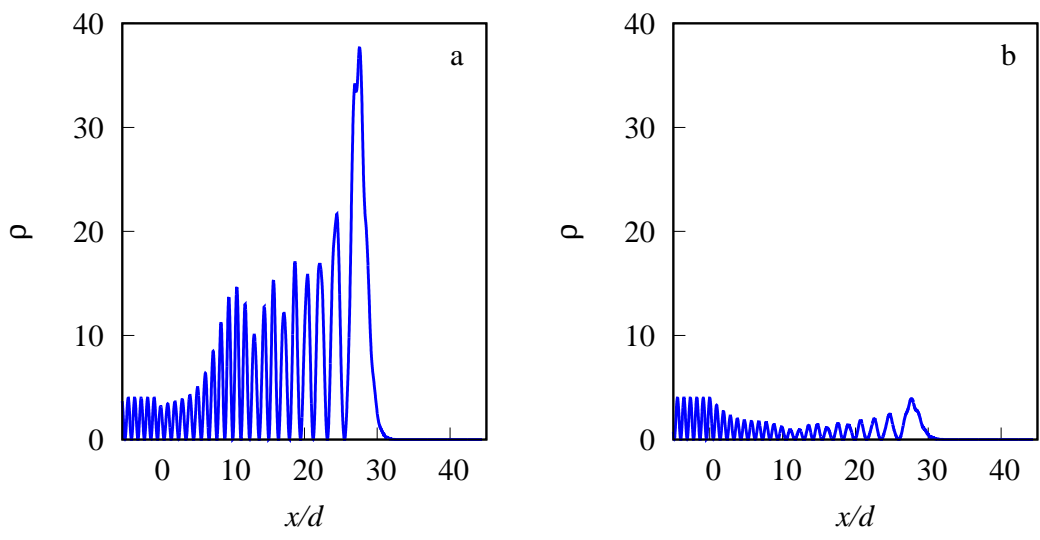

Figure 2: Dependence of probability density $\rho$ on electron $x$-coordinate for $N=40$, $U_{0}=2.5 E_{d}, e F d=0.4 E_{d}$ at $E=12.45 E_{d}(\mathrm{a})$ and $E=12.6 E_{d}(\mathrm{~b})$.

reflection are shown in Fig. 2. The probability density is normalized to unit amplitude of the incident wave.

One can see that depending on energy total electron reflection can be accompanied by a significant increase in $\rho(x)$ (Fig. 2 a.) or can occur without any growth of $\rho(x)$ (Fig. 2 b.). Relatively small change in energy can lead to a significant change in the maximal value of $\rho(x)$. The large values of $\rho(x)$ can be interpreted as long time of electron staying at a given place and can be considered as an indirect attribute of the partial localization.

For the further analysis we have investigated the dependence of maximal probability density $\rho_{\max }$ on the electron energy $E$. One can see from Fig. 3 that in certain intervals of energy $\rho_{\max }$ oscillates as a function of $E$. The period of the oscillations tends to the distance $e F d$ between the Stark levels when the energy approaches the value $N e F d$. The amplitude of the oscillations can be used as the criterion of partial localization. We note that in the case of total reflection the probability density always reaches the value of 4 if the amplitude of incident wave equals unity. That is why the amplitude of oscillations in Fig. 3 is determined mostly by the maximal value of peak on the dependence $\rho_{\max }(E)$. 


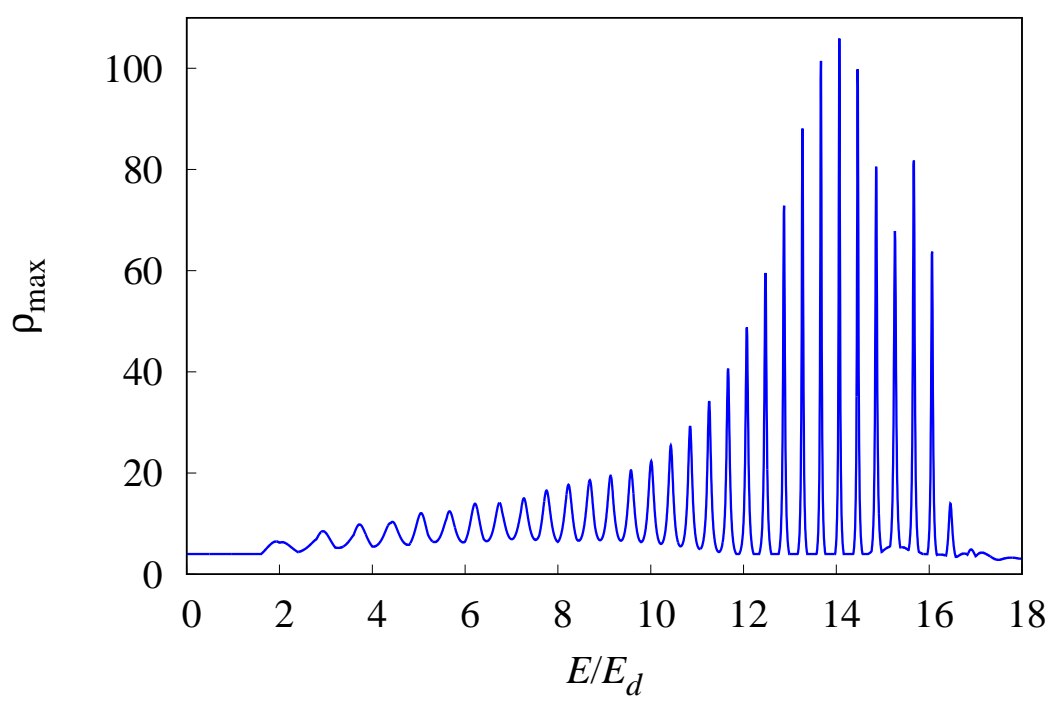

Figure 3: Dependence of maximal probability density $\rho_{\max }$ on electron energy $E$ for the same parameters as in Fig. 2.

At the next step we consider the dependence of the maximal oscillation amplitude on parameters $F$ and $U_{0}$. For this reason we found global maximum of $\rho$ as a function of $x$ and $E$ for given values of $U_{0}$ and $F$. The dependence of $\rho_{\max }$ on the superlattice potential $U_{0}$ at fixed electric field $F$ is shown in Fig. 4. The maximal amplitude of probability density increases very quick with increase in potential barrier $U_{0}$. Thus the localization can be clearly seen in the case of high potential barriers (weak coupled superlattices).

One could expect that the amplitude of oscillations should increase monotonically with $F$ if the electric field is the main reason of the electron localization. However, this is not true. The dependence of maximal probability density $\rho_{\max }$ on electric field is shown in Fig. 5. One can see that the amplitude of probability oscillations increases with electric field in low fields but decreases in higher fields.

Fig. 6] shows the dependence of $\rho_{\max }$ on both parameters $F$ and $U_{0}$. One can see that maximal probability density increases with increase in height 


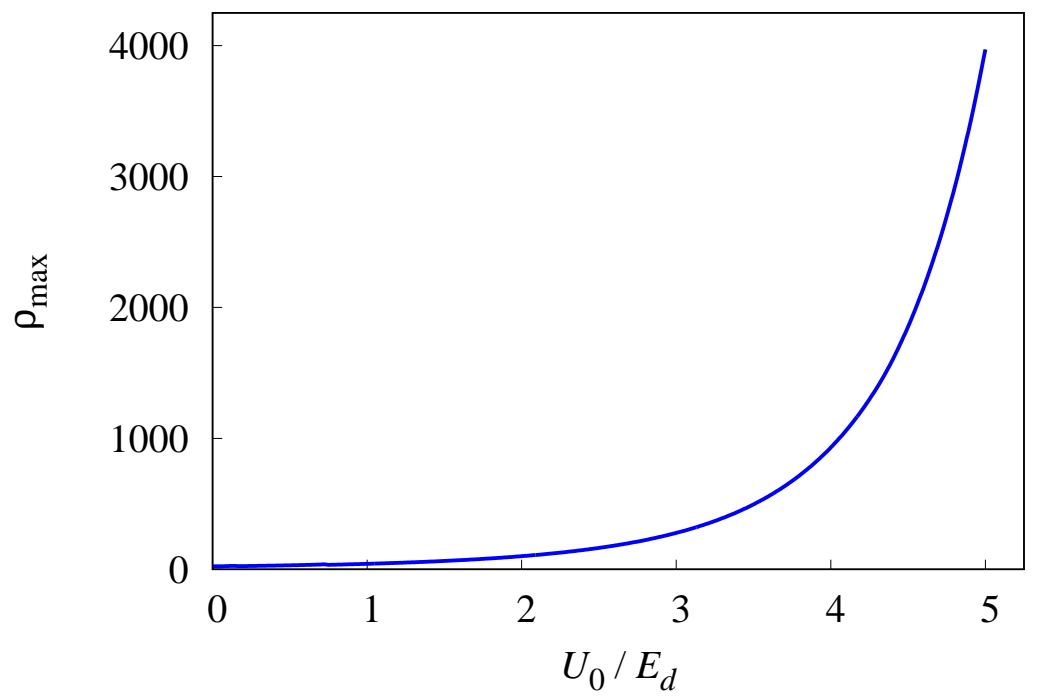

Figure 4: Dependence of maximal probability density $\rho_{\max }$ on the amplitude $U_{0}$ of the superlattice potential for $N=50, e F d=0.3 E_{d}$ and $a=0.4 d$.

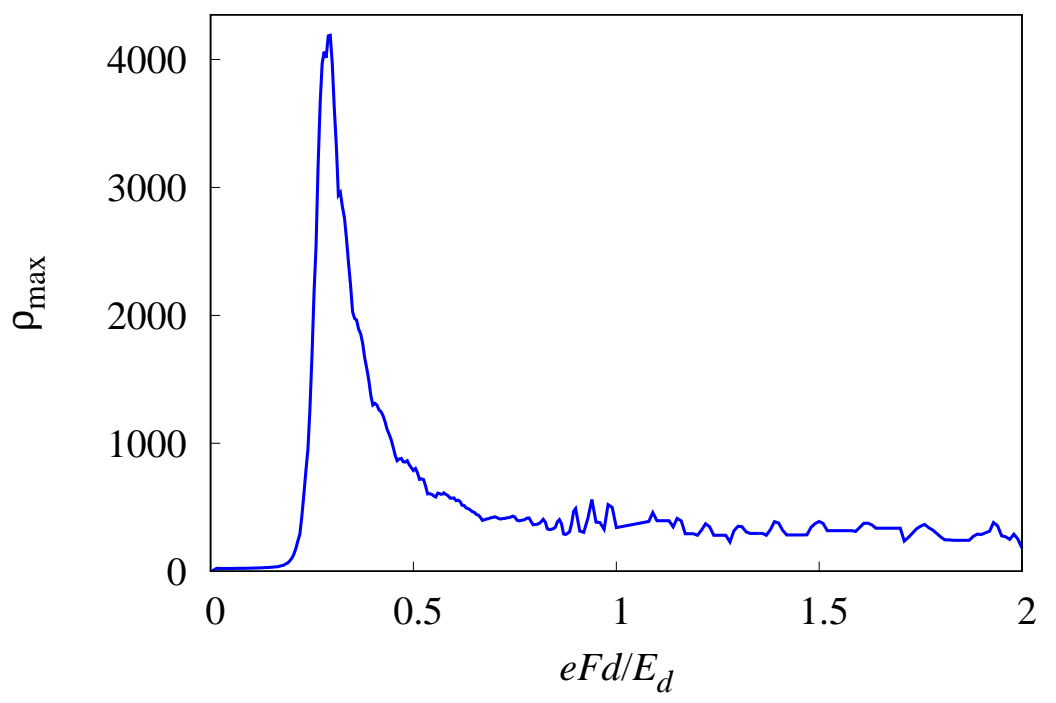

Figure 5: Dependence of maximal probability density $\rho$ on the electric field $F$ for $N=50$, $U_{0}=5 E_{d}$ and $a=0.4 d$. 


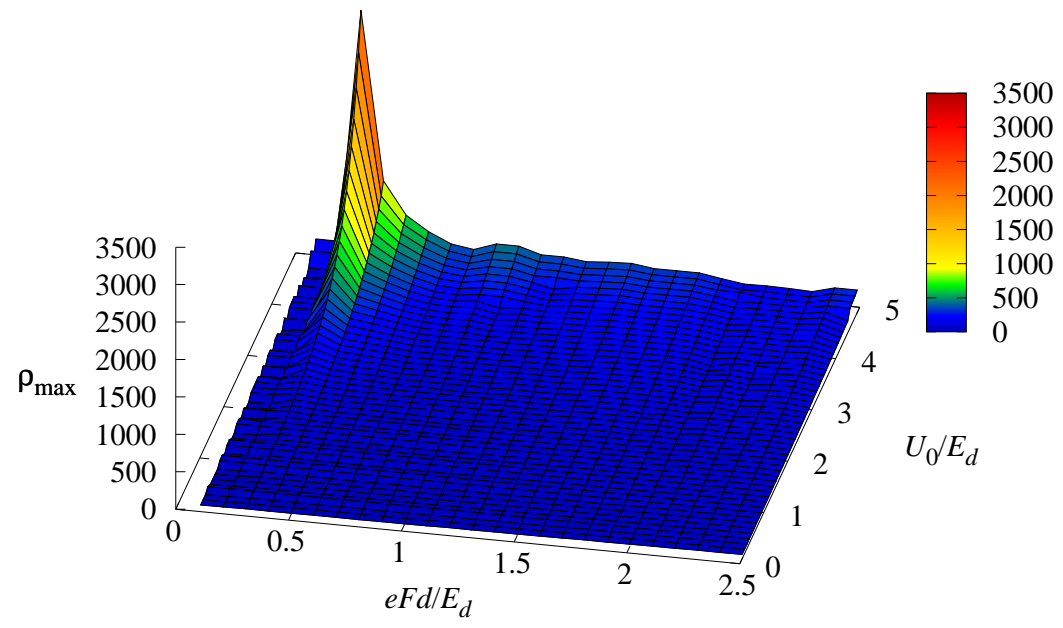

Figure 6: Dependence of maximal probability density $\rho_{\max }$ on potential barrier height $U_{0}$ and electric field $F$ for $N=50, a=0.4 d$.

$U_{0}$ of the potential barrier. At the same time, the dependence of $\rho_{\max }$ on $F$ is non-monotonic that means that the increase in field does not obligatorily lead to higher degree of localization.

\section{Conclusions}

So we can conclude that in the finite-size superlattice the amplitude $U_{0}$ of potential difference plays more important role in formation of the quasilocalized electron states than the electric field. The necessary condition for observation of Wannier-Stark resonances is low coupling between neighbouring quantum wells in the superlattice. On the other hand, there is some "optimal" electric field for every superlattice which makes the resonances most clearly seen.

\section{Acknowledgements}

The work has been supported by the RFBR (grant no. 17-02-00969). 


\section{References}

[1] G. H. Wannier, Phys. Rev. 117, 432 (1960).

[2] J. Zak, Phys. Rev. Lett. 20, 1477 (1968).

[3] J. Zak, Phys. Rev. B 43, 4519 (1991).

[4] J. E. Avron, J. Zak, A. Grossmann, and L. Gunther, J. Math. Phys. 18, 918 (1977).

[5] G. Nenciu, Rev. Mod. Phys. 63, 91 (1991).

[6] M. Glück, A. R. Kolovsky, H. J. Korsch, and F. Zimmer, Phys. Rev. B 65, 115302 (2002).

[7] M. Glück, A. R. Kolovsky, and H. J. Korsch, Phys. Rep. 366, 103 (2002).

[8] E. E. Mendez, F. Agullo-Rueda, and J. M. Hong, Phys. Rev. Lett. 60, 2426 (1988).

[9] P. Voisin, J. Bleuse, C. Bouche, S. Gaillard, C. Alibert, and A. Regreny, Phys. Rev. Lett. 61, 1639 (1988).

[10] E. E. Mendez and G. Bastard, Phys. Today 46, 34 (1993).

[11] G. Tackmann, B. Pelle, A. Hilico, Q. Beaufils, and F. Pereira dos Santos, Phys. Rev. A 84, 063422 (2011).

[12] G. J. Ferreira, M. N. Leuenberger, D. Loss, and J. C. Egues, Phys. Rev. B 84, 125453 (2011)

[13] H. K. Kelardeh, V. Apalkov, and M. I. Stockman, Phys. Rev. B 90, 085313 (2014).

[14] Q. Beaufils, G. Tackmann, X. Wang, B. Pelle, S. Pelisson, P. Wolf, and F. Pereira dos Santos, Phys. Rev. Lett. 106, 213002 (2011).

[15] A. Maury, M. Donaire, M.-P. Gorza, A. Lambrecht, and R. Guérout, Phys. Rev. A 94, 053602 (2016).

[16] V. I. Sankin, Semiconductors 36, 7, 717 (2002). 
[17] V. I. Sankin, A. V. Andrianov, A. O. Zakharin, and A. G. Petrov, JETP Letters 94, 362 (2011).

[18] V. I. Sankin, A. V. Andrianov, A. O. Zakhar'in, and A. G. Petrov, Appl. Phys. Lett. 100, 111109 (2012).

[19] K. A. Ivanov, A. G. Petrov, M. A. Kaliteevski, and A. J. Gallant, JETP Lett. 102, 796 (2015).

[20] W. W. Lui and M. Fukuma, J. Appl. Phys. 60, 1555 (1986).

[21] K. F. Brennan and C. J. Summers, J. Appl. Phys. 61, 614 (1987). 